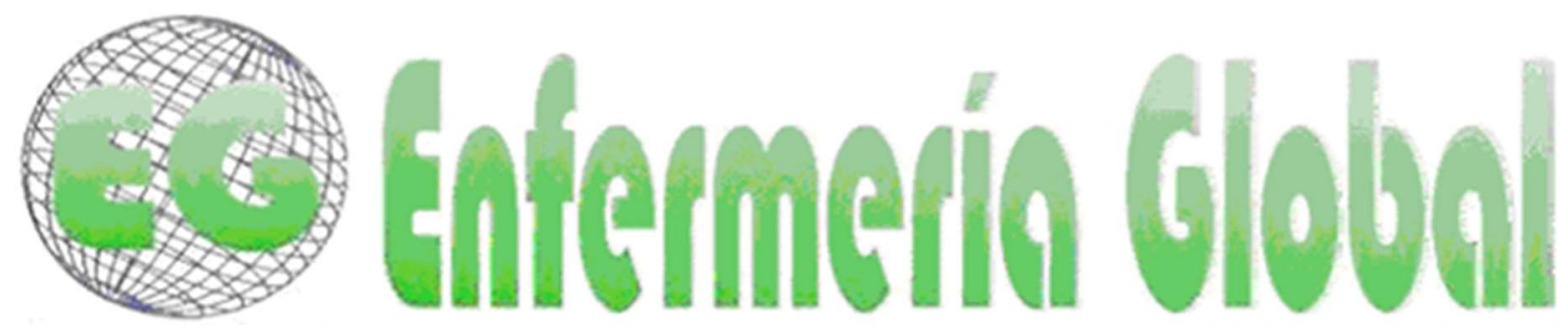

\title{
ORIGINALES
}

\section{Riesgo de diabetes mellitus tipo 2 y sus determinantes}

Risk of type 2 diabetes mellitus and its determinants

Mariel Heredia-Morales ${ }^{1}$

Esther C. Gallegos Cabriales ${ }^{2}$

${ }^{1}$ Estudiante de Doctorado en Ciencias de Enfermería de la Facultad de Enfermería, Universidad Autónoma de Nuevo León. México. marielherediam@gmail.com

2 PhD, Profesora de la Facultad de Enfermería, Universidad Autónoma de Nuevo León. México.

\section{https://doi.org/10.6018/eglobal.482971}

Recibido: 9/06/2021

Aceptado: 14/09/2021

\section{RESUMEN:}

Objetivo: Describir la relación de los determinantes sociales de salud con el riesgo de DMT2 en población mexicana.

Métodos: Estudio descriptivo correlacional transversal, con una muestra de 256 individuos de una comunidad rural de Sinaloa, México. La recolección de datos se realizó durante octubre de 2020 y febrero de 2021. El muestreo fue no probabilístico por bola de nieve. Los instrumentos utilizados fueron el cuestionario internacional de actividad física (IPAQ) versión corta, IPAQ-A, IPAQ-C y una hoja de registro datos sociodemográficos, antropométricos y clínicos.

Resultados: Los indicadores de riesgo de DMT2 con mayor frecuencia para adultos fue padecer hipertensión arterial $(81.7 \%)$ y SP/OB $(68.6 \%)$ y para menores de edad fue tener SP/OB $(34.9 \%)$. Resultó que el riesgo de DMT2 se acrecentaba según lo hacía la edad $(r=.560, p<.01)$ pero disminuía al aumentar la escolaridad de las personas $(r=-.127, p<.05)$.

Conclusiones: El abordaje de factores de riesgo de DMT2 bajo la perspectiva de los DSS brinda la oportunidad de plantear estrategias de salud que contemplen factores contextuales simultáneos al estilo de vida que refuercen las acciones del personal de salud para contribuir a la reducción de los índices de morbimortalidad causados por la DMT2.

Palabras clave: Determinantes sociales de salud; riesgo de diabetes mellitus tipo 2; mexicanos.

\section{ABSTRACT:}

Objective: Describe the relationship between social determinants of health and risk of type 2 diabetes mellitus in Mexican population.

Methods: This was a cross-sectional descriptive correlational study of a sample of 256 individuals from a rural community in Sinaloa, Mexico. Data collection was carried out from October 2020 to February 2021. A snowball non-probability sampling method was used. The Instruments used were the International Physical Activity Questionnaire (IPAQ), short version, the IPAQ-A for adults, the IPAQ-C for children, and a sociodemographic, anthropometric, and clinical data sheet.

Results: The most frequent risk indicators for T2DM for adults are hypertension $(81.7 \%)$ and overweight/obesity $(68.6 \%)$; in children, it was overweight/obesity $(34.9 \%)$. The risk of T2DM increased according to age $(r=.560, p<.01)$ but decreased as education level increased $(r=-.127, p<.05)$ 
Conclusions: The approach to T2DM risk factors from the perspective of social determinants of health allows strategic healthcare planning that considers the contextual factors associated with a lifestyle that reinforces the actions of healthcare providers.

Keywords: Social determinants of health; type 2 diabetes mellitus risk; Mexicans

\section{INTRODUCCIÓN}

La diabetes mellitus (DMT2) es un problema de salud pública que afecta a todo el mundo cuyo impacto aún es más severo en países en vías de desarrollo donde la brecha en las oportunidades de salud está profundamente marcada. La DMT2 está dentro de las primeras diez causas de mortalidad en el mundo, con un aumento del $70 \%$ desde el año $2000{ }^{(1)}$. La prevalencia mundial de DMT2 es del $9.3 \%$, en 2019 murieron más de cuatro millones de adultos y anualmente los casos en niños y adolescentes siguen en aumento ${ }^{(2)}$. En México el panorama no es distinto, según datos oficiales del 2018 la prevalencia de DMT2 en 2018 fue del 10.3\% y es la tercera causa de muerte en el país ${ }^{(3)}$. Aunado a lo anterior se estima que el porcentaje de individuos con DMT2 sin diagnosticar es de poco más del $50 \%$ poniendo en relieve la necesidad del diagnóstico oportuno que permita una atención adecuada para retrasar potenciales secuelas de la enfermedad a nivel individual, familiar y en la sociedad en general $^{(1)}$.

Enfermedades crónicas no transmisibles (ECNT) como la DMT2 son patologías de gran complejidad y representan un desafío para la sociedad y los sistemas de salud en el mundo. El aumento en la prevalencia mundial de DMT2 se ha atribuido a un complejo conjunto de factores socioeconómicos, demográficos y ambientales, así también al aumento de los factores de riesgo para el desarrollo de la enfermedad relacionados con estilos de vida no saludables como sobrepeso/obesidad y bajos niveles de actividad física. La DMT2 requiere una comprensión profunda que considere además de los factores biológicos de las personas, el contexto social en el que se desarrollan las mismas ${ }^{(1)}$.

Los determinantes sociales de salud (DSS) brindan la oportunidad de abordar la DMT2 desde una perspectiva más amplia. Los DSS es una corriente que nació de la necesidad de considerar condiciones de salud más allá del nexo biopsíquico humano en el que se desarrollan enfermedades de gran impacto social como la DMT2. La Organización Mundial de la Salud (OMS) a través de la Comisión de Determinantes Sociales en Salud (CDSS) creó el Modelo de Determinantes Sociales en Salud (MDSS) que muestra como las inequidades en salud están distribuidas en determinantes estructurales e intermedios que afectan la salud y bienestar de la población ${ }^{(4)}$. Así también que las desigualdades en salud tienen una causa social que las precede y origina ${ }^{(5)}$.

Los DSS son un conjunto de circunstancias sociales, políticas, económicas y biológicas en los que los individuos y grupos nacen, crecen y se desarrollan a lo largo de su vida. Se componen de determinantes estructurales e intermedios. Los determinantes estructurales son un complejo bloque socioeconómico político que determinan las condiciones de salud de un país. Mientras que los determinantes intermedios incluyen factores biológicos, comportamentales, genéticos, estilo de vida y psicológicos que los individuos experimentan en cada etapa del ciclo de vida ${ }^{(4)}$. 
La DMT2 es una enfermedad compleja, en cuya aparición interactúan distintos factores sociodemográficos (determinantes estructurales) y comportamentales (determinantes intermedios). Existe evidencia científica que relacionan los DSS con la DMT2; por un lado, se encuentran los determinantes estructurales como ser mujer ${ }^{(6)}$, tener más de 45 años ${ }^{(7)}$ y la no realización de detecciones de enfermedades crónicas no transmisibles (ECNT) ${ }^{(8)}$. Por otro lado, están los determinantes intermediarios como baja actividad física ${ }^{(9)}$, índice de masa corporal $(I M C) \geq 25^{(10)}$ y bajo acceso a servicios de salud (11). La necesidad de considerar variables estructurales e intermedias en la aparición de la patología sigue siendo de relevancia para el área de la salud. En este sentido se plantea la interrogante ¿cuál es la relación de los determinantes sociales de salud con el riesgo de DMT2 en población mexicana?

\section{MÉTODOS}

\section{Diseño, muestra y muestreo}

Estudio descriptivo correlacional transversal. La muestra total corresponde 256 individuos de una comunidad rural de Sinaloa, México, encuestados durante octubre de 2020 y febrero de 2021. El muestreo fue no probabilístico por bola de nieve.

Los criterios de selección de la muestra fueron individuos que contaran con teléfono celular o fijo con la función básica de recepción de llamadas. El criterio de eliminación fue personas con diagnóstico de DMT2, sea por manifestación verbal o por tipo de medicamento y personas menores de seis años.

\section{Recolección de datos}

Primero se seleccionó una población con rezago social medio y bajo ${ }^{(12)}$ que permitiera hacer comparaciones entre ellas. Después de se realizó un recorrido sistemático por la comunidad para identificar puntos de afluencia de personas (ej. tiendas, iglesias) y distribuir anuncios físicos en el que se invitaba a participar en el estudio; simultáneamente se hizo la misma invitación vía digital en la red social Facebook para tratar de llegar a la mayor población posible. El anuncio contenía el objetivo de la investigación y el número de contacto de los investigadores para que el potencial participante hiciera una llamada pérdida, mensaje de texto o de WhatsApp y se pudieran establecer el primer contacto.

El reclutamiento de los participantes se inició con la primera llamada por parte del investigador hacia el primer participante. Los cuestionarios correspondientes y la hoja de datos sociodemográficos, antropométricos y clínicos fueron aplicados por vía telefónica y llenados físicamente por los investigadores. Al finalizar la encuesta se les pidió de favor hicieran llegar a terceros la invitación para crear una respuesta en cadena que permitiera llegar a más personas.

\section{Instrumentos y mediciones}

Se aplicó el instrumento cuestionario internacional de actividad física (IPAQ) (13) versión corta para mayores de 15 años y dos versiones de éste para adolescentes y niños: IPAQ-A (14) para adolescentes de 12 a 14 años de edad e IPAQ-C (15) para niños de 6 a 11 años de edad respectivamente. También se aplicó una hoja de 
registro datos sociodemográficos, antropométricos y clínicos. Todos los instrumentos y mediciones se realizaron únicamente por vía telefónica debido a las restricciones provocadas por la pandemia COVID 19.

EI IPAQ es un cuestionario compuesto de siete ítems que evalúa la actividad física según intensidad (leve, moderada y severa), frecuencia (días) y duración (horasminutos). La frecuencia y la duración se responden anotando el número de días por semana y horas-minutos por día en que realizó la actividad física. El cálculo se realizó de manera automática en una hoja de Excel predeterminada que brinda la página oficial de IPAQ (www.ipap.ki.se). Las puntuaciones más altas indican a mayor nivel de actividad física. En las propiedades psicométricas del instrumento se evaluó la fiabilidad mediante test-retest, obteniendo un coeficiente de correlación $r=.76$ (IC 95\%: .73-.77)(16).

EI IPAQ-A es un cuestionario que pregunta acerca de la frecuencia de actividades físicas que realiza el adolescente en los últimos siete días. Contiene 25 ítems y tiene una escala de respuesta tipo Likert. La puntación final del cuestionario oscila de 1 a 5 puntos y resulta de la media de los ítems. Las puntuaciones más altas indican mayor nivel de actividad física. Respecto a las características psicométricas reportadas para el instrumento, la confiabilidad fue mediante Alpha de Cronbach fue de .83 y la validez mediante un coeficiente de correlación interclase de .73 (IC $95 \%)^{(14)}$.

EI IPAQ-C es prácticamente idéntico al IPAQ-A arriba descrito, incluyendo puntuación e interpretación. La única diferencia es que al IPAQ-C se le agrega una pregunta que cuestiona acerca de las actividades físicas que realiza el niño durante el recreo. Las características psicométricas del IPAQ-C fueron confiabilidad Alpha de Cronbach de .74 y la validez fue analizada mediante un análisis confirmatorio factorial en donde las cargas factoriales fueron de .26 a $.72^{(15)}$.

En la hoja de registro datos sociodemográficos y antropométricos se recolectó datos de edad, sexo, escolaridad, actividad/empleo, realización del programa de detección de DMT2, realización del programa de detección de SP/OB, afiliación a servicios de salud y uso de servicios de salud en los últimos tres meses. Las medidas antropométricas fueron autoinformadas. El índice de masa corporal fue calculado con la formula peso/talla*talla y clasificado según los estándares de la OMS.

\section{Variables}

\section{Variable resultado}

Riesgo de DMT2, se construyó un índice de riesgo para evaluar el desarrollo potencial de DMT2 en adultos y menores de edad. Para adultos, constituido por seis factores: IMC; normopeso ( $\leq 24.9)$, sobrepeso/obesidad $(\geq 25)$. Historia de DMT2 en padres y/o hermanos; sí, no. Riesgo según edad; con riesgo ( $\geq 45$ años), sin riesgo (18-44 años). Presencia de hipertensión arterial; sí, no. Antecedentes de ovarios poliquísticos (mujeres en edad reproductiva); sí, no. Antecedentes de productos macrosómicos; sí, no (7). Para menores de edad, integrado por cuatro factores: IMC; normopeso, sobrepeso/obesidad (SP/OB). Historia de DMT2 en padres y/o hermanos; sí, no. Presencia de acantosis nigricans; sí, no. Antecedentes de ovarios poliquísticos en niñas (12 a17 años de edad); sí, no ${ }^{(17)}$. 


\section{Variables explicativas}

Determinantes estructurales. Se integró por dos variables. Realización de detección de DMT2 (adultos $\geq 20$ años); realizada, no realizada. Realización de detección de SP/OB (sin límite edad) es la realización de la detección en el sector público de salud; realizada, no realizada.

Determinantes intermedios. Constituido por tres variables. Actividad física (AF) es la realización de actividades que empleen el sistema musculo-esquelético para la utilización de energía. La AF fue medida a través del IPAQ ( $\geq 15$ años de edad), IPAQ-A (12-14 años de edad) e IPAQ-C (6-11 años de edad); actividad física baja, moderada y alta. Afiliación a servicios de salud es la adscripción al servicio de salud público; si, no. Uso de servicios de salud, es el tipo de servicio de salud al que acudieron en los últimos tres meses; público, privado, no usó.

\section{Covariables}

Las variables categóricas fueron el sexo; hombre, mujer y empleo/actividad; sin salario (estudiante, ama de casa, no trabaja-no estudia, trabaja-estudia), actividades básicas (campo, construcción y trabajo doméstico), empleados asalariados (chofer, electricista, empleado de tienda, fabrica) y profesionistas (profesionistas y jubilados). Las variables continuas fueron escolaridad y edad.

\section{Análisis de datos}

Para el análisis estadístico se utilizó Statistical Package for the Social Science-SPSS (versión 21) en español. La distribución de normalidad de las variables abordadas se determinó empleando la prueba de Kolmogorov-Smirnov. Las correlaciones entre variables se determinaron mediante correlación de Pearson. Las variables categóricas fueron expresadas en frecuencias y porcentajes.

\section{Aspectos éticos}

El estudio se realizó bajo los estándares del Reglamento de la Ley General de Salud en Materia de Investigación para la Salud de México ${ }^{(18)}$ y aprobados por el Comité de Investigación y Comité de Ética e Investigación de la Facultad de Enfermería de la Universidad Autónoma de Nuevo León. El consentimiento informado fue telefónico (audiograbado) para adultos y asentamiento para menores de edad.

\section{RESULTADOS}

Características sociodemográficas. Los resultados corresponden a 256 personas, residentes de una comunidad rural del municipio de Ahome, Sinaloa. La edad promedio fue de 35.75 años $(D E=17.12)$ de los cuales el $50.4 \%(n=129)$ son mujeres; la escolaridad fue de 8.95 años cursados $(D E=3.65)$ equivalente a preparación básica (primaria y/o secundaria). El empleo/actividad más frecuente fue sin salario (estudiante, ama de casa, no trabaja-no estudia, trabaja-estudia) $(46.1 \%, n$ $=118$ ). 
Determinantes estructurales. Se identificó que el $23.8 \%(n=61)$ se realizó de la detección de DMT2 y el $45.3 \%(n=116)$ la detección de SP/OB.

Determinantes intermedios. La AF que predominó fue la de tipo baja con el $40.2 \%$ ( $n=103)$. El 67.6\% $(n=173)$ contaban con afiliación a alguna institución de salud pública y el $66.8 \%(n=177)$ no acudió a algún servicio de salud en los últimos tres meses. Los datos detallados se pueden consultar en la Tabla 1.

Tabla 1: Datos descriptivos de los individuos

\begin{tabular}{|c|c|c|}
\hline Variable & $\mathbf{M}$ & DE \\
\hline Edad & 35.75 & 17.12 \\
\hline \multirow[t]{2}{*}{ Escolaridad } & 8.95 & 3.65 \\
\hline & $\mathbf{n}$ & $\%$ \\
\hline \multicolumn{3}{|l|}{ Sexo } \\
\hline Hombre & 127 & 49.6 \\
\hline Mujer & 129 & 50.4 \\
\hline \multicolumn{3}{|l|}{ Empleo/actividad } \\
\hline $\begin{array}{l}\text { Sin salario (estudiante, ama de casa, no trabaja- } \\
\text { no estudia, trabaja-estudia) }\end{array}$ & 118 & 46.1 \\
\hline $\begin{array}{l}\text { Actividades básicas (campo, construcción y } \\
\text { trabajo doméstico) }\end{array}$ & 58 & 22.7 \\
\hline $\begin{array}{l}\text { Empleados asalariados (chofer, electricista, } \\
\text { empleado de tienda, fabrica) }\end{array}$ & 66 & 25.8 \\
\hline Profesionistas (profesionistas y jubilados) & 14 & 5.5 \\
\hline \multicolumn{3}{|l|}{ Programa de detección de DMT2 } \\
\hline Realizada & 61 & 23.8 \\
\hline No realizada & 145 & 56.6 \\
\hline \multicolumn{3}{|l|}{ Programa de detección de SP/OB } \\
\hline Realizada & 116 & 45.3 \\
\hline No realizada & 140 & 54.7 \\
\hline \multicolumn{3}{|l|}{ Actividad física } \\
\hline Baja & 103 & 40.2 \\
\hline Moderada & 77 & 30.1 \\
\hline Alta & 76 & 29.7 \\
\hline \multicolumn{3}{|l|}{ Afiliación a servicios de salud } \\
\hline Sí & 173 & 67.6 \\
\hline No & 83 & 32.4 \\
\hline \multicolumn{3}{|l|}{ Uso de servicios de salud (últimos tres meses) } \\
\hline Público & 21 & 8.2 \\
\hline Privado & 64 & 25 \\
\hline \multirow[t]{2}{*}{ No usó } & 171 & 66.8 \\
\hline & $(n=256)$ & \\
\hline
\end{tabular}

Fuente: IPAQ versión corta, IPAQ-A, IPAQ-C y una hoja de registro datos sociodemográficos, antropométricos y clínicos.

Variable resultado. Los indicadores de riesgo de DMT2 con mayor frecuencia para adultos fue la presencia de hipertensión arterial $81.7 \%(n=174)$ y SP/OB $68.6 \%(n=$ 
174). El factor de riesgo predominante en menores de edad fue tener SP/OB $34.9 \%$ ( $n$ = 15) (Tabla 2).

Tabla 2: Indicadores de riesgo de DMT2 por grupo de edad

\begin{tabular}{|c|c|c|c|c|c|c|}
\hline \multirow[t]{2}{*}{ Variable } & \multicolumn{2}{|c|}{$\begin{array}{l}\text { Adultos ( } \geq 18 \\
\text { años) }\end{array}$} & \multicolumn{2}{|c|}{$\begin{array}{c}\text { Menores de } \\
\text { edad ( } \leq 17 \\
\text { años) }\end{array}$} & \multicolumn{2}{|c|}{ Total } \\
\hline & \multicolumn{6}{|c|}{ IMC } \\
\hline Normopeso & 67 & 31.5 & 28 & 65.1 & 95 & 37 \\
\hline \multirow[t]{2}{*}{ Sobrepeso/Obesidad } & 146 & 68.5 & 15 & 34.9 & 161 & 63 \\
\hline & \multicolumn{2}{|c|}{$(n=213)$} & \multicolumn{2}{|c|}{$(n=43)$} & \multicolumn{2}{|c|}{$(n=253)$} \\
\hline
\end{tabular}

Historia de DMT2 en padres y/o hermanos

$\mathrm{Si}$

No

\begin{tabular}{lrcccr}
141 & 66.2 & 0 & 0 & 141 & 55 \\
72 & 33.8 & 43 & 43 & 115 & 45 \\
$(n=213)$ & $(n=43)$ & \multicolumn{2}{l}{$(n=253)$}
\end{tabular}

Presencia de hipertensión arterial

$\mathrm{Si}$

$\begin{array}{llllll}174 & 81.7 & \text { NA } & \text { NA } & 174 & 81.7 \\ 39 & 18.3 & \text { NA } & \text { NA } & 39 & 18.3 \\ (n=213) & & & (n=213)\end{array}$

Riesgo según edad

Con riesgo ( $\geq 45$ años)

$\begin{array}{llllll}80 & 37.6 & \text { NA } & \text { NA } & 80 & 37.6\end{array}$

Sin riesgo (18 a 44 años)

$\begin{array}{lllll}133 & 62.4 & \text { NA } & \text { NA } & 133\end{array}$

62.4

$(n=213)$

$(n=213)$

Antecedentes de productos macrosómicos

$\begin{array}{lllllll}\text { Si } & 21 & 19.3 & \text { NA } & \text { NA } & 21 & 19.3 \\ \text { No } & 88 & 80.7 & \text { NA } & \text { NA } & 88 & 80.7 \\ & (n=109) & & & & (n=109)\end{array}$

Antecedentes de ovarios poliquísticos

$\mathrm{Si}$

$\begin{array}{llllll}15 & 13 & 1 & 11.1 & 16 & 13\end{array}$

No

$\begin{array}{lll}100 & 87 \quad 8\end{array}$

$\begin{array}{lll}88.9 & 108 \quad 87\end{array}$

Presencia de acantosis nigricans

$\mathrm{Si}$

$(n=115)$

$(n=9)^{\star *}$

$(n=124)$

No

$\begin{array}{cccccc}\text { NA } & \text { NA } & 4 & 9.3 & 4 & 9.3 \\ \text { NA } & \text { NA } & 39 \quad 90.7 & 39 & 90.7 \\ & & (n=43) & (n=43)\end{array}$

Fuente: Elaborada por los autores.

Nota: $\mathrm{N}=256$ ( $n=213$ adultos, $n=43$ menores de edad). NA: No aplica para el grupo de edad. * Mujeres en edad reproductiva. ${ }^{* *}$ Mujeres de 12 a 17 años de edad. 
En las correlaciones encontradas para variables estructurales se identificó que a mayor edad de los individuos mayor era la realización de la detección de DMT2 ( $r=$ $.286, p<.01)$. El ser mujer se relacionó positivamente con la realización de detección de DMT2 $(r=.161, p<.05)$ y de SP/OB $(r=.166, p<.01)$. Se encontró que a mayor realización de detección de SP/OB, mayor es la realización de la detección de DMT2 $(r=.637, p<.01)$.

En las relaciones para variables intermedias se pudo observar que a medida que aumentaba la edad, mayor era realización de actividad física por parte de los individuos $(r=.145, p<.05)$. Las personas que tenían afiliación medica publica se realizaban con más frecuencia la detección de DMT2 $(r=.190, p<.01)$ y que aquellas que más usaron los servicios de salud se realizaron más la detección de SP/OB ( $r=$ $.168, p<.01)$.

Finalmente resultó que el riesgo de DMT2 se acrecentaba según lo hacía la edad $(r=.560, p<.01)$ pero disminuía al aumentar la escolaridad de las personas $(r=-$ $.127, p<.05)$. Obsérvese la Tabla 3 .

Tabla 3: Correlación de variables sociodemográficas con índice de riesgo de DMT2.

\begin{tabular}{|c|c|c|c|c|c|c|c|c|c|}
\hline Variable & 2 & 3 & 4 & 5 & 6 & 7 & 8 & 9 & 10 \\
\hline 1. Edad & & & & & & & & & \\
\hline 2. Sexo & - & & & & & & & & \\
\hline 3. Escolaridad & .084 & - & & & & & & & \\
\hline 4. Ocupación & $-.332^{* *}$ & $.299^{* *}$ & - & & & & & & \\
\hline $\begin{array}{l}\text { 5. Realización de. } 286^{* *} \\
\text { detección de DMT2 }\end{array}$ & $.161^{*}$ & -.089 & .089 & - & & & & & \\
\hline $\begin{array}{ll}\text { 6. Realización } & \text { de-.042 } \\
\text { detección } & \text { de } \\
\text { SP/OB } & \end{array}$ & $.166^{* *}$ & .039 & -.009 & $.637^{\star \star}$ & - & & & & \\
\hline 7. Actividad física $.145^{*}$ & $-.126 *$ & .099 & $.134^{*}$ & -.030 & -.017 & - & & & \\
\hline 8. Afiliación medica.033 & -.020 & -.029 & -.024 & $.190^{\star *}$ & .077 & -.098 & - & & \\
\hline $\begin{array}{l}\text { 9. Uso los servicios. } 062 \\
\text { de salud }\end{array}$ & .090 & .031 & .056 & .060 & $.168^{* *}$ & .042 & -.016 & - & \\
\hline $\begin{array}{l}\text { 10.Riesgo } \\
\text { DMT2 }\end{array}$ & .054 & $-.127^{*}$ & $.152^{*}$ & $.277^{\star \star}$ & .030 & $.132^{*}$ & .051 & -.003 & - \\
\hline
\end{tabular}

Nota: $N=256$

${ }^{*} p<.05 ;{ }^{* *} p<.01$

\section{DISCUSIÓN}

El riesgo de DMT2 es un problema actual de salud pública que representa una prioridad en los sistemas de salud del mundo. La idenficación de factores de riesgo de DMT2 resulta de gran importancia para evitar las potenciales repercusiones individuales, familiares y sociales que ésta conlleva, su abordaje bajo el contenido de los DSS permite conocer si el contexto socioeconómico y político y biológico comportamental se relaciona riesgo de desarrollo de la enfermedad. En este estudio se encontró que más la mitad de la población no acudió a realizarse las detecciones de DMT2 y SP/OB en el último año (aunque son ofrecidas de manera gratuita por el sector público de salud), lo que pudiera explicarse en parte a que gran cantidad de las 
personas no tenían afiliación médica pública y esto implicaría un costo económico extra en el gasto personal sumado el confinamiento por la pandemia COVID-19 que llevo a muchas personas a no visitar los servicios de salud públicos y privados del país.

Como parte de la estrategia del Estado para reducir los niveles de incidencia de DMT2, en México se implementaron las detecciones de DMT2 y SP/OB de manera gratuita en el sector de salud público, resultados de esta investigación muestran que las detecciones de DMT2 y SP/OB son realizadas con más frecuencia a medida que avanza la edad y que las mujeres son las principales usuarias, coincidiendo con lo reportado por INEGI en $2018^{(19)}$, lo que pudiera atribuirse por una parte a que las los hombres se perciben en menos riesgo de enfermarse que las mujeres ${ }^{(20)}$ lo que los lleva a que acudan con menos frecuencia a las detecciones. Por otro lado, pudiera haber una disparidad en el rol de género; en la población económicamente activa de México el hombre casi duplica la participación económica respecto a la mujer, segregándola a la realización de actividades no remuneradas como las del hogar, permitiendo a la mujer acudir con mayor facilidad a la realización de pruebas de detección de enfermedades ${ }^{(21)}$.

La realización de actividad física es pieza clave en la prevención de DMT2, las recomendaciones internacionales hacen hincapié en que sea realizada por todos los grupos de edad resaltando los beneficios a corto y largo plazo. En esta investigación se encontró que a medida que aumentaba la edad, las personas hacían más actividad física, sin embargo, la actividad física más realizada no era suficiente para llevar una vida saludable, lo que pudiera convertirla en una falsa percepción de salud para las personas poniendo en riesgo su salud.

Distintos autores han mostrado como las inequidades en la afiliación médica y el uso de servicios de salud incide en la salud individual, incluso pueden llevar a la pobreza $(22,23)$. Según las cifras oficiales de nuestro país ocho de cada diez personas tienen acceso a los servicios de salud (18). Parte de lo encontrado en esta pieza, muestra como la afiliación y acceso a los servicios de salud públicos favorece la realización de detecciones de DMT2 y SP/OB, en parte porque estas detecciones se realizan principalmente en el sector público de salud. En este sentido se esperaría una reducción gradual en las tasas de incidencia de DMT2 y SP/OB, sin embargo, estas enfermedades siguen en claro aumento ${ }^{(24)}$. Lo anterior pone en relieve la importancia de adoptar un nuevo enfoque en el abordaje de ECNT, donde el Estado refuerce actividades de prevención y control de las mismas, ubicando al personal sanitario como un medio que transmita evidencia científica reciente a la población en general( $^{(25)}$.

Respecto a los factores de riesgo de DMT2, variables como la edad y la escolaridad han sido anteriormente estudiadas anteriormente ${ }^{(26)}$. Nuestros hallazgos coinciden con lo encontrado, reforzando la idea que el riesgo de DMT2 está íntimamente relacionado con la edad de las personas y que la alta escolaridad actúa como factor protector que retrasa la aparición de la enfermedad y aunque la edad es un factor inmutable, en este sentido, la realización de actividades dirigidas a prevenir la DMT2 tendría que considerar la preparación escolar de los grupos poblaciones, para que la comprensión de la información sea aprovechada al máximo por sus receptores ${ }^{(25)}$. 
Así mismo los altos porcentajes de presencia de hipertensión arterial en adultos y SP/OB en adultos y menores de edad encontrados en esta investigación representan factores predictores de gran peso para la aparición de DMT2 para el futuro ya que la aparición de síntomas clínicos de la DMT2 puede tardar años o puede ser asociada con otras enfermedades.

Finalmente, la principal limitación del estudio fue que el muestreo no probabilístico utilizado afecta la validez externa, en este sentido en la extrapolación los resultados a otras poblaciones, se debe contemplar que haya similitud en las características de la muestra.

\section{CONCLUSIÓN}

El abordaje de factores de riesgo de DMT2 bajo la perspectiva de los DSS brinda la oportunidad de plantear estrategias de salud que contemplen factores contextuales simultáneos al estilo de vida que refuercen las acciones del personal de salud para contribuir a la reducción de los índices de morbimortalidad causados por ECNT como la DMT2. Se concluye que en esta investigación los principales factores de riesgo de DMT2 para adultos fueron padecer hipertensión arterial mientras que el SP/OB es un factor de riesgo compartido por adultos y menores de edad.

\section{REFERENCIAS}

1. Organización Mundial de la Salud. Las diez principales causas de defunción [Internet]. 2021. [Citado 02 mayo 2021]. Disponible en https://www.who.int/es/newsroom/fact-sheets/detail/the-top-10-causes-of-death

2. Federación Internacional de Diabetes. Atlas de la diabetes de la FID [Internet]. [Citado 06 mayo 2021]. 2019. [Citado 02 mayo 2021]. Disponible en https://www.diabetesatlas.org/upload/resources/material/20200302_133352_2406IDF-ATLAS-SPAN-BOOK.pdf

3. Encuesta Nacional de Nutrición y Salud (ENSANUT). Resultados nacionales [Internet]. 2018. [Citado 03 mayo 2021] Disponible en https://ensanut.insp.mx/encuestas/ensanut2018/doctos/informes/ensanut_2018_infor me final.pdf

4. Comisión de Determinantes Sociales en Salud. A conceptual framework for action on the social determinants of health. Social Determinants of Health Discussion Paper 2. WHO Document Production Services. 2010. [Internet]. [Citado 05 mayo 2021]. Disponible en https://apps.who.int/iris/handle/10665/44489

5. Laurell, A. C. La salud-enfermedad como proceso social. [Internet]. 1982. [Citado 09 mayo 2021]. 2(1): 7-25. Disponible https://www.terceridad.net/SP3/Biblio\%20SPIII_2011_I\%20manolo/LaurellLA\%20SALUD\%20ENFERMEDAD\%20COMO\%20PROCESO\%20SOCIAL.pdf

6. De Fronzo R A. From the triumvirate to the ominous octet": a new paradigm for the treatment of type 2 diabetes mellitus. Clinical Diabetology [Internet]. 2009. [Citado 11 mayo 2021]. 10(3): 101-128. Doi 10.2337/db09-9028

7. American Diabetes Association. Standars of care [Internet]. 2021. [Citado 11 mayo 2021]. $\quad$ Disponible https://care.diabetesjournals.org/content/suppl/2020/12/09/44.Supplement_1.DC1 
8. Aparecida M, Horner B, Guerreiro, D. Atención primaria a las personas con diabetes mellitus desde la perspectiva del modelo de atención a las condiciones crónica. Revista Latino América [Internet]. 2017. [Citado 12 mayo 2021]. 25(1). Doi 10.1590/1518-8345.1474.2882

9. Leiva A M, Martínez M A, Petermann F, Garrido-Méndez A, Poblete-Valderrama F, Díaz-Martínez X, Celis-Morales C. Factores asociados al desarrollo de diabetes mellitus tipo 2 en Chile. Nutrición Hospitalaria [Internet]. 2018. [Citado 16 mayo 2021]. 35(2): 400-407. Doi https://dx.doi.org/10.20960/nh.1434

10. Berkowitz S A, Berkowitz T S, Meigs J B, \& Wexler D J. Trends in food insecurity for adults with cardiometabolic disease in the United States: 2005-2012. PloS one [Internet]. 2017. [Citado 17 mayo 2021]. 12(6): e0179172. Doi 10.1371/journal.pone.0179172

11. Pérez Rodríguez A, Berenguer Gouarnaluses M. Algunos determinantes sociales y su asociación con la diabetes mellitus de tipo 2. MEDISAN [Internet]. 2015. [Citado 18 mayo 2021]. 19(10): 1268-1271. ISSN 1029-3019.

12. Consejo Nacional de Evaluación de la Política de Desarrollo Social (CONEVAL). Índice de rezago social 2015 a nivel nacional, estatal y municipal [Internet]. 2015. [Citado 18 mayo 2021] Disponible en https://www.coneval.org.mx/Medicion/IRS/Paginas/Indice_Rezago_Social_2015.aspx 13. International Consensus Group (IPAQ). Cuestionario internacional de actividad física [Internet]. 1996. [Citado 19 mayo 2021]. Disponible en www.ipaq.ki.se

14. Manchola-González J, Bagur-Calafat C, Girabent-Farrés M. Fiabilidad de la versión española del cuestionario de actividad física PAQ-C. Revista Internacional de Medicina y Ciencias de la Actividad Física del Deporte [Internet]. 2017. [Citado 19 mayo 2021]. Doi http://dx.doi.org/10.15366/rimcafd2017.65.008

15. Moore J B, Hanes J C, Barbeau P, Gutin B, Treviño R P, Yin Z. Validation of the Physical Activity Questionnaire for Older Children in children of different races. Pediatric Exercise Science [Internet]. 2007. [Citado 19 mayo 2021]. 19(1): 6-19. Doi 10.1123/pes.19.1.6

16. Mantilla-Toloza S M, Gómez-Conesa A. El Cuestionario Internacional de Actividad Física. Un instrumento adecuado en el seguimiento de la actividad física poblacional. Revista Iberoamericana de Fisioterapia y Kinesiología [Internet]. 2007. [Citado 20 mayo 2021]. 10(1): 48-52. Disponible en https://www.elsevier.es/esrevista-revista-iberoamericana-fisioterapia-kinesiologia-176-articulo-el-cuestionariointernacional-actividad-fisica--13107139. ISSN 1138-6045

17. Norma Oficial Mexicana NOM-015-SSA2-2010. Para la Prevención, Tratamiento y Control de la Diabetes Mellitus [Internet]. 2010. [Citado 19 mayo 2021]. Disponible en: http://www.dof.gob.mx/normasOficiales/4215/salud/salud.htm

18. Reglamento de la Ley General de Salud en Materia de Investigación para la Salud [Internet]. 2014. [Citado 20 mayo 2021] Disponible en http://www.dof.gob.mx/nota_detalle.php?codigo=5339162\&fecha=02/04/2014

19. Instituto Nacional de Estadística Geografía e Informática. Encuesta Nacional de Salud y Envejecimiento en México [Internet]. 2018. [Citado 16 mayo 2021]. Disponible en

https://www.inegi.org.mx/contenidos/saladeprensa/boletines/2020/ENASEM/Enasem_ Nal20.pdf

20. Academia Nacional de Medicina de México. El hombre y la mujer enferman en forma diferente [Internet]. 2014. [Citado 01 junio 2021]. Disponible en http://www.scielo.org.mx/scielo.php?script=sci_arttext\&pid=S0026-

17422014000200053 
21. Encuesta Nacional de Ocupación y Empleo [Internet]. 2021. [Citado 01 junio 2021].

Disponible

en https://www.inegi.org.mx/contenidos/saladeprensa/boletines/2021/iooe/iooe2021_03.p df

22. Balandrán-Duarte D A, Mújica Ó J, Narro-Robles J R, López-Cervantes $M$, Gutiérrez J P, Durán-Arenas J L, Fajardo-Dolci G E. Desigualdad social en México en el uso de servicios de tamizaje en adultos: un análisis de las encuestas nacionales de salud 2006 y 2012. Salud Pública de México [Internet]. 2020. [Citado 03 junio 2021]. 62(5): 511-520. Doi https://doi.org/10.21149/10413

23. Padovani Clemente S, Pazos San Martín, E., Padovani Cantón A M, Ruano Ortega M. Determinantes sociales de la salud y sistema de gestión de la calidad en servicios estomatológicos. Revista Habanera de Ciencias Médicas [Internet]. 2014. [Citado 04 junio]. 13(5): 757-768. ISSN 1729-519X

24. Centro de Investigación en Alimentación y Desarrollo. La pandemia de diabetes en México. Oficina de Prensa y Colaboradores [Internet]. 2020. [Citado 04 junio 2021]. Disponible en https://www.ciad.mx/notas/item/2450-la-pandemia-de-diabetes-enmexico

25. Organización Mundial de la Salud. Enfermedades crónicas y promoción de salud. Prevención de enfermedades crónicas [Internet]. 2021. [Citado 04 junio 2021]. Disponible en https://www.who.int/chp/chronic_disease_report/part1/es/

26. Pinilla A, Barrera M, Rubio C, Devia C. Actividades de prevención y factores de riesgo en diabetes mellitus y pie diabético. Acta médica colombiana [Internet]. 2014. [Citado 04 junio 2021]. 39(3): 250-257. Disponible en http://www.scielo.org.co/pdf/amc/v39n3/v39n3a08.pdf

ISSN 1695-6141

( COPYRIGHT Servicio de Publicaciones - Universidad de Murcia 